\title{
Liquid crystal based tunable WDM planar Bragg grating devices based on precision sawn groove substrates
}

\author{
B. D. Snow ${ }^{1 \dagger}$, J. C. Gates ${ }^{1}$, F. R. M. Adikan ${ }^{1}$, C. B. E. Gawith ${ }^{1}$, A. Dyadyusha ${ }^{2}$, \\ H. E. Major ${ }^{1}$, D. O. Kundys ${ }^{1}$, M. Kaczmarek ${ }^{2}$, and P. G. R. Smith ${ }^{1}$ \\ ${ }^{I}$ Optoelectronics Research Centre, University of Southampton, Southampton, SO17 1BJ, United Kingdom \\ ${ }^{2}$ School of Physics and Astronomy, University of Southampton, Southampton, SO17 1BJ, United Kingdom \\ ${ }^{\dagger}$ bds@orc.soton.ac.uk
}

Current optical telecommunication systems employ dense Wavelength Division Multiplexing (WDM) techniques to increase the data carrying capacity of fiber networks. Dynamic add/drop and filtering processes are crucial for the precise control of individual channels on these networks. Reconfigurable integrated optical devices, such as planar Bragg gratings, can tune the reflection wavelength over several standard channel spacings, providing the possibility for all-optical dynamic networks. Planar devices have the potential to address and tune several channels simultaneously, and have greater potential for integration than fiber equivalents.

We have previously used liquid crystals (LCs) as an adaptive overlayer to tune the Bragg reflection wavelength by exploiting the large electrooptic coefficient of the LC. Electrically tunable planar devices shift the Bragg wavelength by modifying the effective refractive index of a waveguide in a multilayer substrate via LC molecular reorientation. We have determined the electrical tunability range of planar Bragg gratings with a LC overlayer to be $932.7 \mathrm{pm}$ (corresponding to $114 \mathrm{GHz}$ at $\lambda=1561.8 \mathrm{~nm}$ for TE polarization) with $170 \mathrm{Vpp}$ applied across the LC cell [1]. These samples contained channel waveguides with integral Bragg gratings defined using Direct Grating Writing (DGW) [2] and required the removal of the overclad in our 3-layer silica-on-silicon samples via chemical etching. The LC was applied to this etched window to interact with the grating and sealed with an ITO coverslip containing patterned electrodes (Fig.1.a). However, this geometry allows the formation of line discontinuities in the LC bulk, known as disclinations, close to the grating [3]. The wavelength shift observed in these devices is not a linear function of applied field, and hysteresis was seen in the tuning curves.
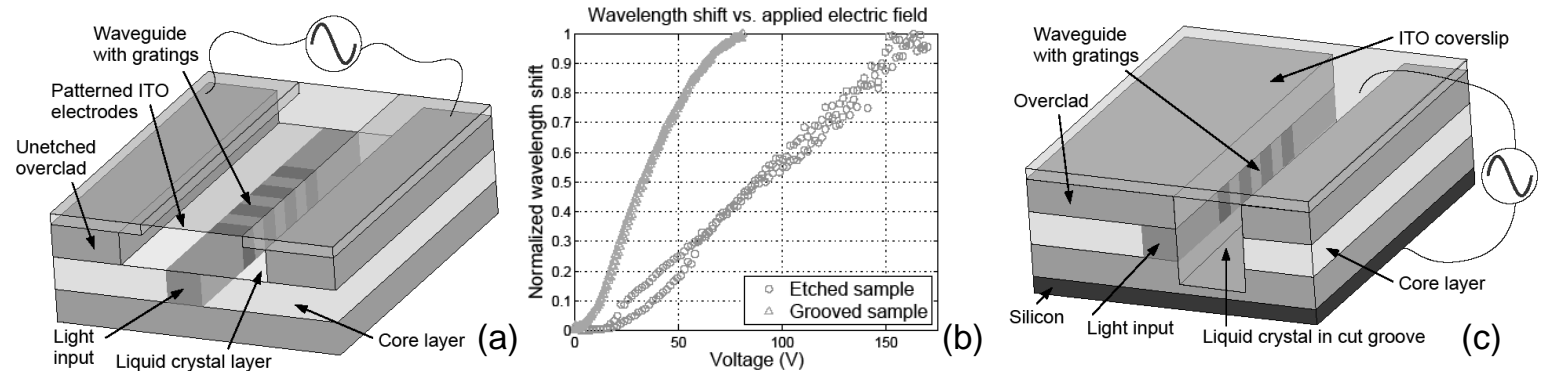

Fig. 1. Illustrations of (a) etched and (c) grooved tuning cells with (b) tuning data for both

To circumvent this non-linear behaviour we have designed and tested an alternative geometry for our tunable devices. Our new groove geometry aims to relocate these disclinations far from the Bragg grating. Thus tuning of the Bragg wavelength should be a smooth function of applied voltage as the LC molecules reorient along the applied field lines. Shown in Fig.1.(c) are our new cells which are easier to fabricate than previous devices as they require no wet etching. These LC cells have grooves cut into the silica using a high precision dicing saw with cutting parameters optimized to give a smooth, high quality wall finish. Subsequently, channel waveguides with gratings can be defined alongside the groove with DGW. This allows the a.c. field to be applied vertically producing a more uniform field across the LC region. The disclinations are therefore confined to regions where the surfactant coated surfaces have perpendicular anchoring directions in close proximity to each other. This restricts the disclinations to the corners of the grooved region, thus preventing the hysteresis seen in etched samples. Fig.1.(b) shows the wavelength shift versus applied electric field for both samples. The etched sample tuning curve contains hysteresis at low voltages, and becomes linear above a threshold. However, for the grooved sample no hysteresis is seen, thus the tuning response is a smooth function of applied field. We will discuss the design of LC tunable devices and how these can be accurately calibrated to provide all-optical dynamic reconfiguration of channels in WDM systems.

\section{References}

[1] F. R. M. Adikan, J. C. Gates, A. Dyadyusha, H. E. Major, C. B. E. Gawith, I. J. G. Sparrow, G. D. Emmerson, M. Kaczmarek, and P. G. R. Smith, "Demonstration of $100 \mathrm{GHz}$ electrically tunable liquid-crystal Bragg gratings for application in dynamic optical networks," Opt. Lett. 32, 1542-1544 (2007)

[2] G. D. Emmerson, S. P. Watts, C. B. E. Gawith, V. Albanis, M. Ibsen, R. B. Williams, and P. G. R. Smith, "Fabrication of directly UVwritten channel waveguides with simultaneously defined integral Bragg gratings," Elec. Lett. 38, 1531-1532 (2002).

[3] B. D. Snow, F. R. M. Adikan, J. C. Gates, C. B. E. Gawith, A. Dyadyusha, M. Kaczmarek, and P. G. R. Smith, "Line defects and temperature effects in liquid crystal tunable planar Bragg gratings," Opt. Express 15, 17129-17135 (2007). 